\title{
Computed tomographic appearance of an oleothorax
}

\section{BENNETT $P$ DEBOISBLANC, WILMOT C BURCH JR, HOWARD A BUECHNER, EDWARD F HAPONIK}

\author{
From the Department of Medicine, Louisiana State University Medical Center, New Orleans, USA
}

\begin{abstract}
Sequelae of oleothorax, formerly used in the treatment of tuberculosis, may still be encountered. A patient is reported whose oleothorax, created 44 years previously, was demonstrated by computed tomography.
\end{abstract}

Instillation of oil into the pleural or extrapleural space to create an oleothorax was often performed during the second quarter of this century as an adjunct to collapse therapy for tuberculosis and for treatment of tuberculous empyema. ${ }^{1}$ The approach was abandoned in the early 1950s with the advent of effective chemotherapy, but patients may still present with unrecognised residua of this regimen.

\section{Case report}

A 73 year old woman presented in January 1985 with slowly progressive exertional dyspnoea of 10 years' duration. In 1941 she was found to have a left tuberculous empyema and was treated with "antiseptic oil." She improved symptomatically and was discharged home for convalescence, but was lost to follow up. Physical examination showed nothing remarkable except for dullness and absence of breath sounds over the left hemithorax. Routine posteroanterior and lateral chest radiographs performed on admission showed dense pleural calcification enclosing what appeared to be a massive left pleural effusion (fig 1). Computed tomography of the chest showed a triphasic layering of the effusion characteristic of an oleothorax (fig 2).

\section{Discussion}

Both vegetable and mineral oils were used to produce an oleothorax and were frequently rendered antiseptic by the addition of gomenol, a volatile product of distillation of the leaves of the myrtle tree. ${ }^{2}$ With collapse therapy up to $2000 \mathrm{~cm}^{3}$ of oil was instilled and left in place for about $18-24$ months while the infected lung was "rested." Over half of the patients, however, developed intense pleuritis associated with fever and prostration that required early removal of the oil. In tuberculous empyema such intense pleural reaction was thought to cleanse the pleural space. Indeed, many patients thus treated improved symptomatically. Some of these

Address for reprint requests: Professor Edward F Haponik, Louisiana State University Medical Center, 1542 Tulane Avenue, New Orleans, LA 70112, USA.

Accepted 12 August 1987 individuals were lost to follow up, as with our patient, and the oil was never removed. Late sequelae have included pleural calcification, bacterial empyema, bronchopleural or $\vec{\omega}$ pleurocutaneous fistula, or expansion. ${ }^{3}$ In a patient reported $V$ by Mulkin and coworkers computed tomography of the chest if helped in the differential diagnosis of these complications. ${ }^{4} \mathrm{~N}$

Our patient's computed tomogram clearly shows layering 음 of the encased effusion. The most radiolucent layer, with a density of -162 Hounsfield units (HU), is also the most buoyant and floats anteriorly in this supine view. This density characterises this layer as pure lipid. The intermediate layer $\vec{\theta}$ has a density of $-43 \mathrm{HU}$, suggesting an emulsion of oil and serous fluid. The most dependent layer has a density of $8 \mathrm{HU}$,

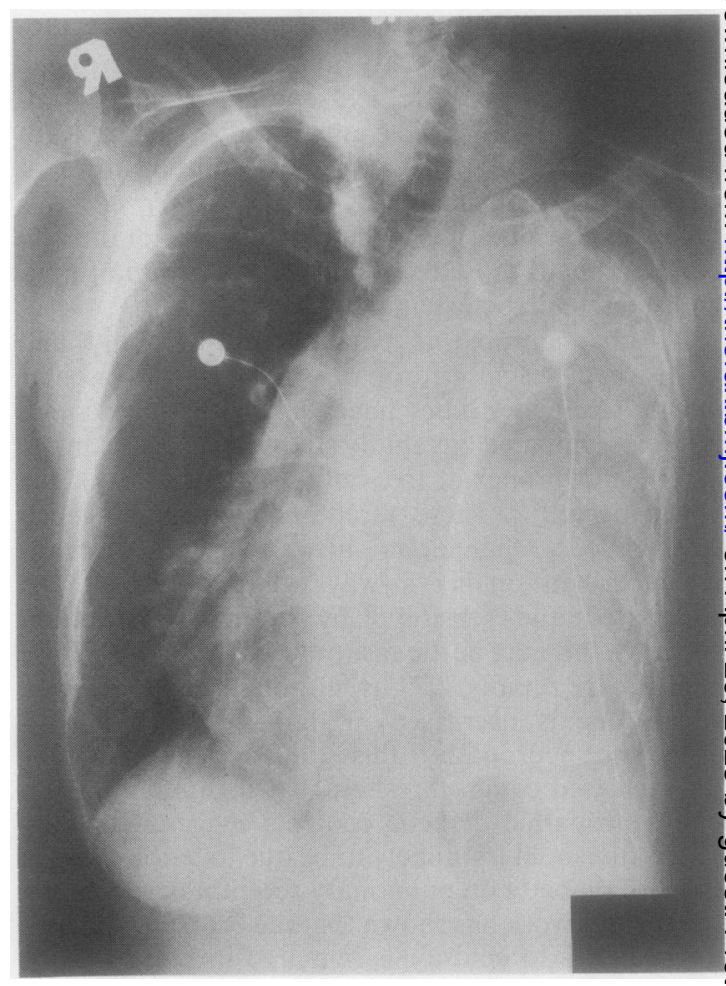

Fig 1 Chest radiograph showing dense pleural calcification surrounding a large left pleural effusion, old fibronodular and cavity infiltrates in the right apex, and a calcified right paratracheal node. 


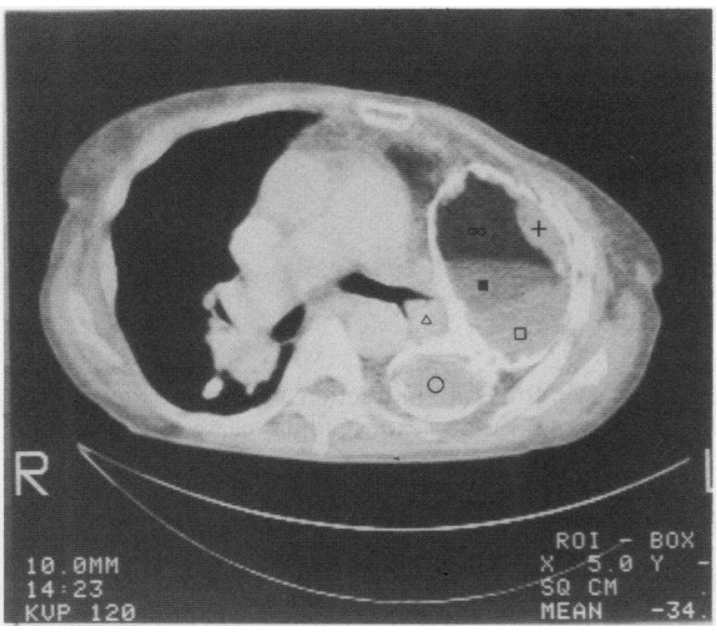

Fig 2 Contrasted chest computed tomography axial image at about the level of $T 7$ showing pleural calcifications enclosing a pleural effusion of three different densities: oil layer $(\infty)$, oil and serous fluid emulsion ( $\square)$, and aqueous cellular suspension ( $\square$ ). Also seen are areas of pleural thickening (+), the left lung compressed against the left hilum to a crescent $(\triangle)$, and a calcified loculation extending posteriorly (O). which is intermediate between that of water $(0 \mathrm{HU})$ and soft tissue (about $36 \mathrm{HU}$ ), and is thus characteristic of an aqueous-cellular suspension. Interestingly, this triphasic appearance of the computed tomogram corresponds well with the layering characteristics observed in the classic investigations of oleothorax of Joannides,' and has not been reported previously. Of the other radiographic findings, the irregular pleural densities are thought to represent areas of pleural thickening from intense chronic inflammation. The left lung is compressed medially, creating a restrictive defect that was the likely cause of dyspnoea in this patient.

\section{References}

1 Joannides $M$. Oleothorax in pulmonary collapse therapy of pulmonary tuberculosis. Am Rev Respir Dis 1936;34:561-613.

2 Matson RW, Goldberg B. Oleothorax. In: Goldberg B, ed. Clinical tuberculosis. Philadelphia: FA Davis, 1947: 161-200.

3 Hutton L. Oleothorax. Expanding pleural lesion. $A J R$ 1984;142:1107-10.

4 Mullin DM, Rodan BA, Bear WJ, Gocke TM, Feng TS. Computed tomography of oleothorax. JComput Tomog 1986;10:197-9. 